\title{
Fluid therapy in paediatric burn injuries - should the current rules be changed?
}

\author{
Jakub Noskiewicz, Olgierd Pietkiewicz, Przemysław Mańkowski \\ Department of Paediatric Surgery, Traumatology, and Urology, Poznan University of Medical Sciences, Poznan, Poland
}

\section{ABSTRACT}

\begin{abstract}
Aim of the study: Tissue damage resulting from thermal injury is associated with systemic disturbances of the water-electrolyte balance. This process consists of exudation and increased evaporation within the burnt area. Therefore, appropriate fluid therapy plays a key role in stabilisation of the general condition and treatment of burns. It is even more important in children with burns. It should be verified whether the existing fluid therapy rules require modification. This is the purpose of the study.

Material and methods: An analysis was performed on hospitalisation of 310 children with isolated burn injury. Hydration rate, followed by electrolyte balance and acid-base balance, were evaluated. Fluid balance and everyday body weight were recorded. The obtained data were analysed using the descriptive statistical methods and tools.

Results: In the group of patients treated according to the Parkland formula, a significant overhydration rate was observed. Overhydration at admission to hospital was recorded in $42.6 \%$ of patients, and after 24 hours of hospitalisation in $59.8 \%$ of children. Children with burn injuries covering up to $20 \%$ of total body surface area (TBSA) required no additional fluid supplementation exceeding the daily fluid intake in order to maintain normal hydration as well as electrolyte and acid-base balances. The sodium bicarbonate solution was not necessary to achieve correction of acid-base balance at the first stage of burn disease management.

Conclusions: Application of technologically advanced dressings results in reduced local fluid escape, which is associated with increased risk of patient overhydration and necessary modification of the commonly applied principles of fluid therapy. Children with burn injuries covering up to $20 \%$ of TBSA do not require supplementation of any other fluids than crystalloids, in particular supplementation with sodium hydrogen carbonate solution in order to maintain the correct value of the water-electrolyte and acid-base balance.
\end{abstract}

KEY WORDS:

burn, fluid resuscitation, Parkland formula, child.

\section{INTRODUCTION}

Tissue damage resulting from thermal injury is associated with systemic disturbances of the water-electrolyte balance. Burn wounds, in particular those covering a significant area of the body surface, contribute to significant fluid loss in children. This process consists of exudation and increased evaporation from the burnt area. Therefore, appropriate fluid therapy, in particular in the first hours after thermal injury, plays a key role in stabilisation of general condition and treatment of burns. It is even more important in children with burns, in whom the percentage body water content is significantly higher than in adults, and who are more susceptible to induction of

\section{ADDRESS FOR CORRESPONDENCE:}

Jakub Noskiewicz, Department of Paediatric Surgery, Traumatology, and Urology, Poznan University

of Medical Sciences, 27/33 Szpitalna St., 60-572 Poznan, Poland, ORCID: 0000-0001-5473-0824,

e-mail: jnoskiewicz@op.pl 
systemic disturbances caused by excessive fluid loss via damaged tissues.

The existing advanced dressings have significantly changed treatment of burn wounds. On one hand, these dressings create a humid environment improving the healing rate. On the other hand, their occlusive component significantly reduces fluid loss via damaged tissues decreasing exudation and slowing down the evaporation rate. However, the most commonly applied fluid therapy rules are still based on the Parkland formula developed by Charles R. Baxter in the Parkland Memorial Hospital in Dallas in the 1960s, where burn treatment was associated with high fluid loss and its necessary supplementation [1]. At the same time, application of the advanced occlusive dressings and of fluid resuscitation formula not adjusted to decreased exudation and evaporation poses a risk of water-electrolyte disturbances in patients with burn injuries - in particular during the first 24 hours of treatment. This risk is most severe in the youngest patients. Therefore, it should be verified whether the existing fluid therapy rules require modification.

\section{MATERIAL AND METHODS}

An evaluation of 310 paediatric patients hospitalised for burn injuries in the Department of Paediatric Surgery, Traumatology, and Urology of Poznan University of Medical Sciences was performed. All patients up to 18 years of age hospitalised in 2010-2017 for an isolated thermal injury were enrolled in the study. The main cause of injury was contact with hot liquid within the upper body half, covering on average $10.0 \%$ of total body surface area (TBSA), provided that the patient with the most extensive thermal injury had $55.0 \%$ of TBSA injured. In order to avoid measurement error, the evaluation of burnt body surface was performed independently by two health professionals, members of the medical team. Second-degree (partial thickness) burns were dominating.

In the first part of the research, a group of 275 patients with isolated burn injuries was evaluated retrospectively. These patients were hospitalised in the years 2010-2016 and treated strictly according to the Parkland formula for children (Table 1). Crystalloids, primarily multi-electro-

TABLE 1. Parkland formula for children on the first day of treatment

\begin{tabular}{|l|l|}
\hline & Fluid formula \\
\hline Day 1 & $4 \mathrm{ml} \times$ total body surface area of burn $(\%) \times$ body \\
& weight $(\mathrm{kg})$ : \\
& - first half of the solution over the first 8 hours \\
& - second half of the solution over the next 16 hours \\
& + \\
& baseline fluid intake: \\
& $4 \mathrm{ml} / \mathrm{kg} / \mathrm{h}$ for 10 first $\mathrm{kg}$ of body weight plus \\
& $2 \mathrm{ml} / \mathrm{kg} / \mathrm{h}$ for 10 second $\mathrm{kg}$ of body weight plus \\
& $1 \mathrm{ml} / \mathrm{kg} / \mathrm{h}$ for $>20$ first $\mathrm{kg}$ of body weight \\
\hline
\end{tabular}

lyte fluid or Ringer's solution, were used for fluid therapy. Water-electrolyte and acid-base balances were evaluated at admission to the surgery department and after 24 hours of hospitalisation as well as at discharge from hospital. Fluid balance and everyday body weight measurements were recorded. All patients were treated according to the same therapeutic scheme, according to which damaged tissues, upon surgical treatment, were covered with hydrocolloid occlusive dressing.

In the second part of the research, new fluid therapy recommendations were developed. According to the accepted rules, patients were given a volume of fluids, which resulted from their baseline daily intake (described in Table 1). The preferred route was oral supply, but $1 / 3$ of the baseline fluid intake was administered intravenously during the first two days of treatment. Only crystalloids, primarily multi-electrolyte fluid or Ringer's solution, were used for fluid therapy. New fluid therapy recommendations were prospectively verified on the same structural group of 35 patients with isolated burn injuries hospitalised in the year 2017. No exclusion criteria were applied. Water -electrolyte and acid-base balances were also evaluated. Fluid balance and everyday body-weight measurements were also recorded. All patients were treated according to the same therapeutic scheme as in the first group, i.e. the same: wound treatment, analgesic therapy, therapy of shock, and nutritional treatment.

Additionally, during the retrospective part of the study, fluid therapy control cards for children with burn injury were introduced. In these control cards, the patient's hydration status, volume of fluids administered intravenously and taken orally by the burned child, and changes in the child's body weight were recorded in detail. The impact of increased control of fluid therapy (without changing the rules of fluid therapy) on the hydration status was evaluated.

The obtained data were analysed using descriptive statistical methods and tools, adopting a significance level of $p<0.05$. Consent to perform this study was obtained from the Bioethical Committee at Poznan University of Medical Sciences (no. 175/17).

\section{RESULTS}

In the analysed group of 275 patients treated according to the Parkland formula, a significant overhydration rate was observed. Haematocrit values below the lower limit of the standard at admission to hospital were recorded in $42.6 \%$ of the patients, whereas in $59.8 \%$ of children after 24 hours of hospitalisation, provided that the haematocrit drop between the zero (mean: $35.0 \pm 2.9 \%$ ) and first (mean: $33.2 \pm 3.1 \%$ ) day of hospitalisation was of statistical significance $(p=0.0001)$. The average blood sodium concentrations in the zero $(136.0 \pm 2.9 \mathrm{mmol} / \mathrm{l})$ and first $(136.7 \pm 3.5 \mathrm{mmol} / \mathrm{l})$ day of hospitalisation fell within the laboratory standards. The average blood po- 
tassium concentration at the initial stage of treatment was $4.2 \pm 0.5 \mathrm{mmol} / 1$. After 24 hours of therapy it was still in the laboratory standard $(4.5 \pm 0.6 \mathrm{mmol} / \mathrm{l})$. All children were discharged from hospital with normalised water-electrolyte balance as well as acid-base balance, which was observed in the performed control laboratory tests at discharge.

A gradual, spontaneous correction of acid-base balance without the need for balancing its disturbances by supplying $8.4 \%$ sodium bicarbonate solution at the first stage of burn disease management was recorded. Supply of $8.4 \%$ sodium bicarbonate solution in the treatment of burn diseases was required only in one patient. Exceeding the limit value of base excess (BE) at the start of hospitalisation was recorded in $43.9 \%$ of children, but this was insignificant. After the next 24 hours, this proportion amounted to $33.7 \%$. Average BE values at the first stage of hospitalisation did not deviate from the laboratory standards and manifested a fractional upward trend (-2.6 $\pm 2.0 \mathrm{mEq} / \mathrm{l}$ in zero day vs. $-1.7 \pm 1.9 \mathrm{mEq} / \mathrm{l}$ after 24 hours of therapy), provided that this trend was statistically significant $(p=0.0076)$ (Table 2).

Considerable disproportions in the volume of intravenously administered and total volume of administered fluids at the initial stage of treatment of paediatric patients treated according to the Parkland formula were observed, i.e. at the time of the greatest risk of water-electrolyte disturbances. Intravenously administered fluid volume during the first 24 hours of therapy ranged between $0.5 \%$ and $15.8 \%$ of body weight of the patient. It correlated with the extensiveness of damaged tissues $(p=0.0350)$. The total volume of fluids administered within the first 24 hours of treatment did not exceed $17.0 \%$ of body weight. Similarly shaped values were recorded in the second day of treatment (Fig. 1). Excessive fluid supplementation was associated with body weight increase. In extreme cases a body weight increase by $25.0 \%$ in 24 hours was recorded. In the cases of weight gain during the first 48 hours of burn treatment, an increase in the children's weight by more than $10.0 \%$ was recorded (mean: $11.9 \% \pm 9.3 \%$ ). Overhydration required administration of diuretics only in rare cases. The vast majority of the patients normalised their water-electrolyte disturbances spontaneously by eliminating excessive fluid via urine, without the need for introduction of pharmacotherapy. In addition, some patients even tolerated - without any effect on body weight and haematocrit change - a daily supply of fluids reaching up to $16.7 \%$ of body weight (half of which was supplemented intravenously). The overhydration in the first two days of treatment did not affect the hospitalisation period $(p=0.3510)$.

Introduction of fluid therapy control cards for the patients with burn injuries (without changing the rules of fluid therapy) resulted in a better control of the conducted fluid therapy. These control cards facilitated the assessment of the fluid balance and limited the latitade in fluid
TABLE 2. Fluid, electrolyte, and acid-base balance on admission to the hospital and after 24 hours of treatment according to the Parkland formula (mean and standard deviation)

\begin{tabular}{|l|c|c|}
\hline & Admission & After 24 h \\
\hline Haematocrit & $35.0 \pm 2.9 \%$ & $33.2 \pm 3.1 \%$ \\
\hline Sodium & $136.0 \pm 2.9 \mathrm{mmol} / \mathrm{l}$ & $136.7 \pm 3.5 \mathrm{mmol} / \mathrm{l}$ \\
\hline Potassium & $4.2 \pm 0.5 \mathrm{mmol} / \mathrm{l}$ & $4.5 \pm 0.6 \mathrm{mmol} / \mathrm{l}$ \\
\hline Base excess (BE) & $-2.6 \pm 2.0 \mathrm{mEq} / \mathrm{l}$ & $-1.7 \pm 1.9 \mathrm{mEq} / \mathrm{l}$ \\
\hline
\end{tabular}

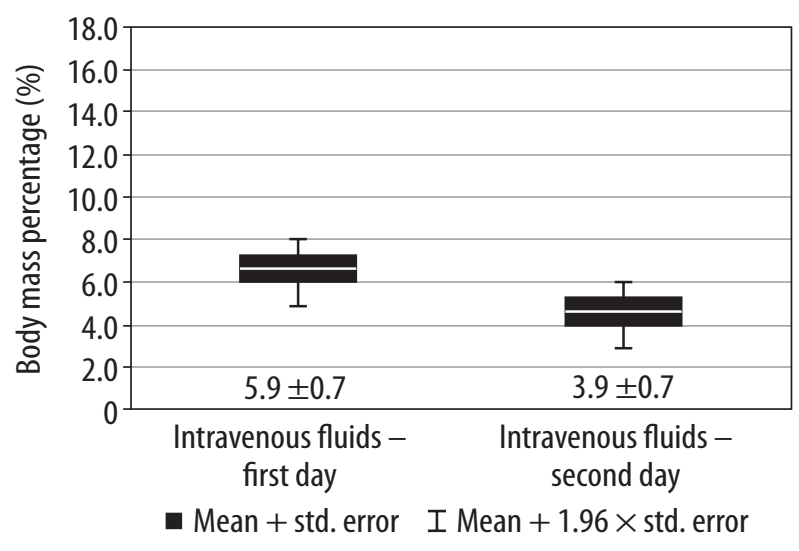

FIGURE 1. Intravenously administered fluid volume on the first and second days of treatment according to the Parkland formula - percentages in relation to the patient's weight

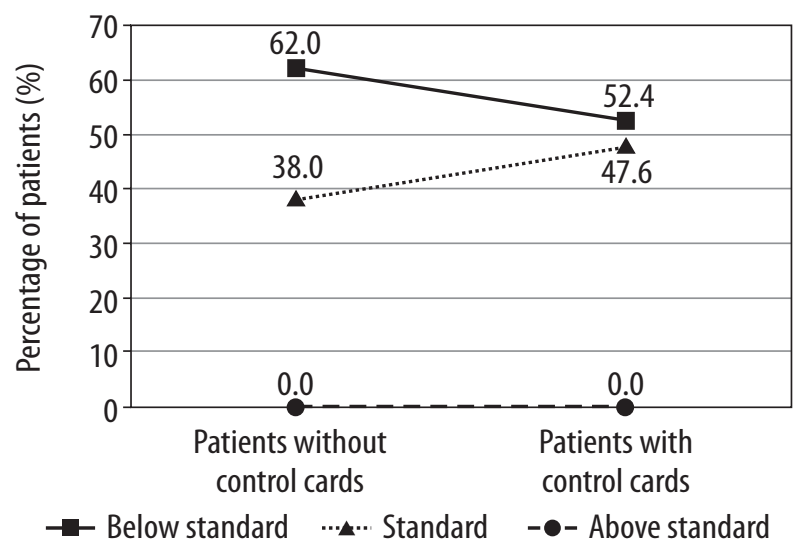

FIGURE 2. Influence of introduced fluid therapy control card on the haematocrit value evaluated after 24 hours of hospitalisation

therapy. As a result, a decrease in the number of children with reduced haematocrit was observed after 24 hours of hospitalisation (from $62.0 \%$ to $52.4 \%$ ) (Fig. 2).

In the case of paediatric patients with burn injuries covering up to $20 \%$ of TBSA, a simple correlation between the volume of fluids administered intravenously within the first 24 hours of hospitalisation and haematocrit value $(p=0.0057)$ and its change in this period ( $p=0.0205)$ was recorded. At the same time, it was noticed that these patients required no additional fluid supplementation exceeding the daily fluid intake in order to maintain normal hydration as well as electrolyte and ac- 
id-base balances. Part of the daily fluid requirement had to be administered intravenously via infusion of crystalloids. In the studied group, this volume accounted for $1 / 3$ of baseline fluid intake. The effectiveness of this procedure was verified prospectively on a group of 35 patients with a positive effect - no significant water-electrolyte or acid-base balance disturbances were observed. Haematocrit did not deviate from the norm. Overhydration was not observed.

Similar regularities were not recorded in the patients with burn injuries covering more than $20 \%$ of TBSA. In this group, both the scope and method of fluid resuscitation required individual determination.

\section{DISCUSSION}

An adequate fluid resuscitation is undeniably one of the key elements of the therapeutic procedure that should be introduced in patients with burn wounds. There is a great deal of evidence that both excessive and insufficient fluid supplementation have a negative effect on the treatment process and even lead to increased mortality. In addition, fluid demand in children with small and medium-size burn injuries is difficult to estimate. Considering the fact that the burns in the vast majority of paediatric patients cover up to $20 \%$ of TBSA, the issue of adequate volume of fluid resuscitation becomes of significance [2-5].

The issue of overhydration of a child with burns occurs as early as at the stage of transport to the hospital. In the material under study, approximately half of the patients were admitted to the surgery department after supplementation with excessive volume of infusion fluids. The above can partially result from incorrect estimation of the size of the burn by the medical rescue team or casualty department personnel and therefore incorrect calculation of fluid demand. This problem was brought up by Hagstrom et al., who evaluated fluid resuscitation in adults with burn injuries before transferring the patients to the burn treatment centre. In the population under study, an overestimated surface of damaged tissues and hence excessive fluid therapy was identified in $30.0 \%$ of patients. At the same time, the researchers emphasise that early contact with a burn specialist and collaborative development of a therapeutic scheme could eliminate such errors. Similar observations, however, on a group of paediatric patients were made by Goverman et al., provided that in their study excessive fluid therapy was applied to $59.0 \%$ of children. Lindford et al. demonstrated that the use of burn charts, which are currently available as phone apps, facilitated calculation of fluid demand and increased the correctness of calculations by $20.0 \%$. Similar conclusions can be found in the publications by Bodger et al. or by Williams and Doerfler. Dingley et al. presented the opportunities of applying the advanced technologies to solve the issue of fluid resuscitation, the introduction of which is associated with a statistically lower risk of error. In the population under study, introduction of fluid therapy control cards for the patients with burn injuries decreased the number of over-hydrated children by approximately $10.0 \%$, primarily by improving calculation of the size of burn [6-11].

The issue of poorly planned fluid resuscitation is not only associated with incorrect estimation of the size of the burn, which - as demonstrated by the studies of Parvizi et al. - applies also to specialists treating patients with thermal injuries on an everyday basis. The reason behind this problem is also, perhaps even primarily, continuous use of the Parkland formula or the other fluid therapy formulas, including the paediatric ones developed on its basis. The vast majority of the currently used technologically advanced dressings decrease fluid escape via the wound and in effect require decreased volume of administered fluids. At the same time, the importance of fluid hypoalimentation as one of the burn management components is increasingly frequently emphasized. Hollén et al. point out a positive impact of reducing the volume of fluids administered to children with burns covering $10-20 \%$ of body surface area. Similar conclusions are drawn by the authors of this publication. In addition, Walker et al. state that application of decreased fluid resuscitation reduces the need for skin graft and length of hospital stay. Similar results were not obtained in this study, which probably results from correctly functioning compensation capacity and the ability to immediate reduce overhydration among paediatric subjects. At present, the other methods of fluid therapy planning and management, which are based on repeatable analysis of water - electrolyte balance (Pulse index Contour Continuous Cardiac Output - PiCCO or photoplethysmography) are being assessed [2, 3, 5, 12-14].

The preferred type of infusion fluids should also be determined. Balanced crystalloids are used commonly and with satisfactory results. In most cases, the supply of sodium hydrogen carbonate solution in order to balance minor acid-base disturbances is unnecessary, which is confirmed by the results presented in this study. On the other hand, there is evidence confirming the effectiveness of the other formulas used in fluid resuscitation, in particular in severe burns. Kao et al. point out the positive effects of the use of hyperosmotic agents for this purpose, while Fodor et al. describe the effective treatment examples based on a combination of crystalloids and plasma supply, provided that the initial fluid resuscitation is based on crystalloids $[4,15]$.

The authors of this publication evaluated the fluid therapy in children with burn injuries covering up to $20.0 \%$ of TBSA, in detail. These subjects constitute the essential and baseline patient population. Therefore, specification of the fluid therapy rules in this group seems to be of particular importance. The obtained results confirm that when the burn wounds are appropriately dressed, supplementation of additional fluid volume above the 
baseline daily intake is not required. The route of administration remains, however, a separate issue. The preferred route is oral supply, although reluctance of a child to drink immediately after injury and the need for temporary restraining from fluid supply, for example due to surgical procedures or supply of anaesthetics, imposes partially intravenous fluid therapy. Therefore, initial intravenous administration of crystalloids in a volume constituting $1 / 3$ of the baseline fluid intake, followed by adjusted fluid therapy in the presence of exacerbating drinking reduction or visible water-electrolyte or acid-base disturbances in the case of burns covering up to $20 \%$ of TBSA, seems to be optimal.

\section{CONCLUSIONS}

Application of technologically advanced dressings results in reduced local fluid escape, which is associated with the increased risk of patient overhydration and necessary modification of the commonly applied principles of fluid therapy.

Children with burn injuries covering up to $20 \%$ of TBSA do not require supplementation of any fluids other than crystalloids, in particular supplementation with sodium hydrogen carbonate solution in order to maintain the correct value of the water-electrolyte and acid-base balance.

\section{DISCLOSURE}

The authors declare no conflict of interest.

\section{REFERENCES}

1. Baxter CR, Shires T. Physiological response to crystalloid resuscitation of severe burns. Ann N Y Acad Sci 1968; 150: 874-894.

2. Kraft R, Herndon DN, Branski LK, et al. Optimized fluid management improves outcomes of pediatric burn patients. J Surg Res 2013; 181: 121-128.

3. Noskiewicz J, Rzanny-Owczarzak M, Mańkowski P. Paediatric burn injuries - retrospective evaluation of applied therapeutic management. Pediatr Pol 2018; 93: 433-437.

4. Fodor L, Fodor A, Ramon Y, et al. Controversies in fluid resuscitation for burn management: literature review and our experience. Injury 2006; 37: 374-379.

5. Stewart CL, Mulligan J, Grudic GZ, et al. A noninvasive computational method for fluid resuscitation monitoring in pediatric burns: a preliminary report. J Burn Care Res 2015; 36: 145-150.

6. Hagstrom M, Wirth GA, Evans GR, Ikeda CJ. A review of emergency department fluid resuscitation of burn patients transferred to a regional, verified burn center. Ann Plast Surg 2003; 51: 173-176.

7. Goverman J, Bittner EA, Friedstat JS, et al. Discrepancy in Initial Pediatric Burn Estimates and Its Impact on Fluid Resuscitation. J Burn Care Res 2015; 36: 574-579.

8. Lindford AJ, Lim P, Klass B, et al. Resuscitation tables: a useful tool in calculating pre-burns unit fluid requirements. Emerg Med J 2009; 26: 245-249.
9. Bodger O, Theron A, Williams D. Comparison of three techniques for calculation of the Parkland formula to aid fluid resuscitation in paediatric burns. Eur J Anaesthesiol 2013; 30: 483-491.

10. Williams D, Doerfler R. Graphic aids for calculation of fluid resuscitation requirements in pediatric burns. Ann Plast Surg 2012; 69: 260-264.

11. Dingley J, Cromey C, Bodger O, Williams D. Evaluation of 2 novel devices for calculation of fluid requirements in pediatric burns. Ann Plast Surg 2015; 74: 658-664.

12. Parvizi D, Kamolz LP, Giretzlehner M, et al. The potential impact of wrong TBSA estimations on fluid resuscitation in patients suffering from burns: things to keep in mind. Burns 2014; 40: 241-245.

13. Hollén L, Coy K, Day A, Young A. Resuscitation using less fluid has no negative impact on hydration status in children with moderate sized scalds: a prospective single-centre UK study. Burns 2017; 43: 1499-1505.

14. Walker TL, Rodriguez DU, Coy K, et al. Impact of reduced resuscitation fluid on outcomes of children with 10-20\% body surface area scalds. Burns 2014; 40: 1581-1586.

15. Kao Y, Loh EW, Hsu CC, et al. Fluid Resuscitation in Patients with Severe Burns: A Meta-Analysis of Randomized Controlled Trials. Acad Emerg Med 2018; 25: 320-329. 